\title{
Optimization of Intraday Trading Strategy Based on ACD Rules and Pivot Point System in Chinese Market
}

\author{
Xue Tian ${ }^{1}$, Cong Quan ${ }^{2}$, Jun Zhang ${ }^{3}$, H. J. Cai ${ }^{3}$ \\ ${ }^{1}$ School of Economics and Management, Wuhan University, Wuhan, China; ${ }^{2}$ College of Software Technology, South China Agricul- \\ tural University, Guangdong, China; ${ }^{3}$ International School of Software, Wuhan University, Wuhan, China. \\ Email: quancong121@hotmail.com,jim.zoumo@foxmail.com,hydra6@gmail.com \\ Received May $15^{\text {th }}, 2012$; revised July $3^{\text {rd }}, 2012$; accepted July $10^{\text {th }}, 2012$
}

\begin{abstract}
Various trading strategies are applied in intraday high-frequency market to provide investors with reference signals to be on the right side of market at the right time. In this paper, we apply a trading strategy based on the combination of ACD rules and pivot points system, which is first proposed by Mark B. Fisher, into Chinese market. This strategy has been used by millions of traders to achieve substantial profits in the last two decades, however, discussions concerning on the methods of calculating specific entry point in this trading strategy are rare, which is crucial to this strategy. We suggest an improvement to this popular strategy, providing the calculating and optimizing methods in detail to verify its effectiveness in recent Chinese futures market. Because of the high liquidity and low commissions in stock index futures market, this trading strategy achieves substantial profits. However, given the less liquidity in commodity futures market, profits decrease and even be neutralized by the relatively high commissions.
\end{abstract}

Keywords: ACD Rules; Pivot Point System; Pivot Range; Optimization

\section{Introduction}

China has experienced rapid development on stock index and commodity futures market in recent years, and various studies concerning on high frequency data to achieve profits are springing out, discussions of which range from arbitrage opportunity [1] to wave theory [2]. Accurate predictions of price movements in futures market will bring large profits in trading, which has become a challenging task for investors and a focus for academicians. Quantitative methods and techniques have been widely applied to forecast price trend. The Polynomial Classifiers and back propagation feed forward neural networks models were conducted on Dubai Financial Market to forecast securities' prices movements [3]. Regression and Neural Network models are used for predicting Shanghai Composite Index returns and price volatility [4]. More models such as ARMA-GARCH, recurrent SVM and recurrent RVM are also applied for forecasting [5]. V. Pacelli did deep empirical studies on Artificial Neural Network to forecast exchange rates and credit risk management [6,7], and also developed this model for further research [8]. Various kinds of Neural Network models are famous for their advantages of adaptability to complex situations, suiting to reveal hidden relationships that govern the data. However, Neural Network models still subject to the risk of local minima, especially in models with more three layers. The data ratio for model training and forecasting is always set empirically, unable to realize automatically renewal. The model we introduce in this paper deals with this limits well, as the combination of parameters, including data ratio for training, is renewed in time according to market data to seek optimums continually and maximize profits.

In this paper, we introduce an improvement to a popular intraday trading strategy based on ACD rules and the pivot point system. The basic premise of the strategy is that the price range and trend established during the first few minutes of market open sets the tone for the rest of the day in the sense of making high and low price for the full day already observable, with much greater likelihood than chance. ACD rules trading strategy was first created by Mark. B Fisher, a famous American professor of trend trading. It provides entry and exit points for long or short intraday. Mark. B Fisher used this trading method for more than 20 years and introduced it to the public in his book "The Logic Trader: applying a method to the madness" [9].

Among most investors who had applied it to their trading system, their profits proved its effectiveness. Since then, many related studies concerning with this method comes out $[10,11]$. Moreover, part of this trading strat- 
egy, the pivot point system can derivate various complicate technique analysis [12,13], including prevail 5 points system, 7 points system and 13 points system. Now we only care about the pivot range in this system to combine with the ACD rules to forecast the formation of price trend as well as mood and atmosphere of the market, providing investors with reference entry and exit points.

In ACD rules, we usually choose the high and low price of the first 5 - 20 minutes as the upper and lower bounds of opening range. Aup or Adown point is on or below the bounds of opening range for a specific distance. When the price stays above Aup point for half of the time domain of opening range, the Aup point is established successfully and the stop-loss point B is the lower bound of opening range in this case. After Aup point has been established, the reverse entry point is C. It is the turning point of the market atmosphere, from bullish to bearish or from bearish to bullish. It indicates a strong change in market trends, providing investors with signal about how to trade. It is away from the bounds of opening range for some larger distance than that of A. After Aup point has been established, if the price falls strongly instead of increasing, reaching Cdown point, then investors should establish a clear preference for short at this time, seizing the market downward trend to get profits as much as possible. In this case, the stop-loss point, $\mathrm{D}$, is one tick above the upper bound of opening rang. Situation on the other side of the market is symmetric.

The other part of this trading strategy, pivot point system, is mainly used to determine the price support or resistance zone. The relative places of these zones and the last close price forecast the market mood of next day. Combining both in trading, double signals from them increase investors' confidence to maximize positions to get more profits. It is also helpful to refine the stop-loss point $\mathrm{B}$ and $\mathrm{D}$ to minimize loss.

However, In the process of implementing this trading strategy, the most crucial and difficult problem for each investor is how to determine the right place of entry point A and C. In 2010, a report from Guosen applied the trading strategy that based on ACD rules and pivot point system into stock index futures in Chinese market and realized substantial excess profits [14]. However, it fails to talk about methods for calculating the place of $\mathrm{A}$ and $\mathrm{C}$, which is unique for each future in different market. In this paper, we focus on improving this strategy, discussing how to determine the place of these two important entry points, which are determined by two important parameters. We program this trading strategy to find the optimal combination of parameters. In empirical study, we apply this auto-trading system into stock index and commodity futures market in China to prove its effectiveness.

\section{An Empirical Example}

We choose a commodity future, SR1209, to show how to apply this method to intraday trading in details. Take the commodity futures SR1209, on Jan 5th, for example. We take the average of the previous 4 transaction days' range (high-low), and use $12 \%$ of this value as the distance between Aup/Adown and the upper/lower bound of the opening range. On Jan 5th, the highest and lowest prices of the first 20 minutes are 6202 and 6102 respectively. So the opening range of this transaction day is 6102 6202, r2 - r1 in Figure 1(a).

The pivot range (not present in figures) in pivot point system is calculated by the high $(\mathrm{H}), \operatorname{low}(\mathrm{L})$ and close price $(\mathrm{C})$ of the previous one transaction day, namely Jan 4th.

Specific formulas are as follows: (PP: pivot price; SV: the second value; PD: pivot difference; PR: pivot range)

$$
\begin{aligned}
& \mathrm{PP}=(\mathrm{H}+\mathrm{L}+\mathrm{C}) / 3 \\
& \mathrm{SV}=(\mathrm{H}+\mathrm{L}) / 2 \\
& \mathrm{PP}-\mathrm{SV}=\mathrm{PD} \\
& \mathrm{PP}+/-\mathrm{PD}=\mathrm{PR}
\end{aligned}
$$

Figure 1(a) shows that the price breaks Aup level at 9:57. From then on, the program starts to calculate time. Once the price stays above Aup level for more than 10 minutes, it will prompt to open long positions.

Then the time goes to 10:06 and the price has stayed above Aup level for just 10 minutes. It will prompt investors to open long positions ("upa" point in Figure 1(a)). The price of this moment is 6228 . Since then, the program will judge the point where to close the long positions. If the price rises continually, then investors can sell them after 10 ticks rise. According to history data of sugar trading, we set tick as 1 . Of course, the amount of tick may not be fixed. It varies in accordance with price range of certain futures and investors preference However, if price falls. Instead of increasing, investors should close the long position at stop-loss point. Combing ACD rules and the pivot point system, the stop-loss point B in this case is the higher one between the lower bound of opening range, 6028 and that of pivot range, 6102. So B is 6102 in this example. If neither of these above two situations occurs, then close them at the closing time, 15: 00.

From Figure 1(a), we see that price rises continually, reaching 6243, more than 10 ticks, at 10:17. Then investors can close the long positions at this moment. Calculating profits by formula: (sell price-buy price)/sell price, we get $0.241 \%$ gains.

Later, the price stays above A up level for the remaining time of this day, the program will not prompt to open long positions or short positions, for it is unlikely for price to have another significant rise. However, if price 
falls below A up point, and goes above it, staying for sufficient time again, it will prompt to open long positions once more.

Next, in Figure 1(b), we give another example, CU1204 on Feb 1st, to show how to deal with the situation on the other side of the market. The price falls below A down level and stay below it for 10 minutes at 11:24. The program will prompt to open short positions at this place, price of which is 59,730 .

Then the price decreases continually for more than 10 ticks at 11:27. Investors can close the short positions at this place by price of 59,680, getting profits. After this trading, we can see that the price have a small increase. But it falls below A down level and stay below it for 10 minutes once more at 13:57. Investors can open short positions again at this place and close them later.

Then, we can see clearly from trend that, it has no opportunity today after two transactions. Thus profit of this day is $0.15 \%$ in total.

\section{Optimization}

We apply this trading strategy to stock index and commodity futures market in China to prove its effectiveness. The core of this auto-trading system is to find the right place of entry point $\mathrm{A}$ and $\mathrm{C}$ precisely. From the example in Part2, we see that the place of A point for different futures are determined by two major parameters, the

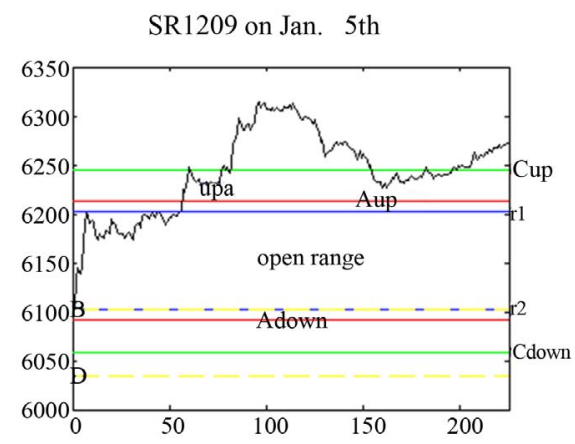

(a)

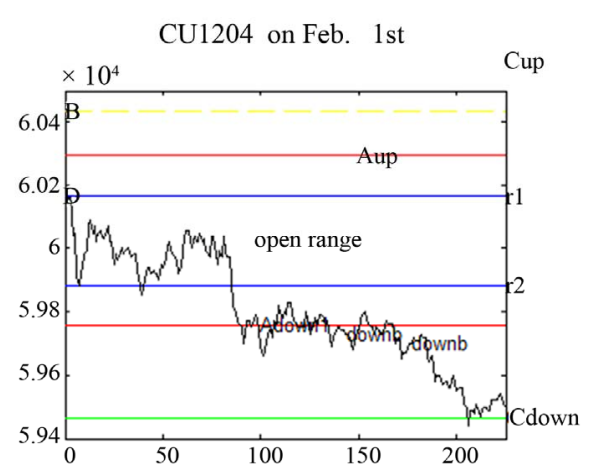

(b)

Figure 1. Trading process. number of the past transaction days we take to get average range, called $n$, and the percent of that value we use, called P. Since C is the turning point of market mood, trade opportunity occurring at point $\mathrm{C}$ is so rare that it has little influence on profits. In our study, we set the distance between $\mathrm{C}$ up/down and the upper/lower bound of the opening range two times of that of $\mathrm{A}$.

In this trading model, we need high frequent price data of the first 20 days to train this model, getting the optimal combination of parameters, $n$ and $P$. They will be used to calculate the proper place of entry point A for the next day (See Figure 2).

For training model, we select all combinations of $n$ and $\mathrm{P}$ within their effective range and calculating profits of each combination as approach mentioned in Part 2. All these results gathered, we draw them in one 3 dimension draft to look for the highest point in the direction of profits. The corresponding coordinate is the optimal combination of $\mathrm{n}$ and $\mathrm{P}$.

Here, we take IF1206 in stock index futures market as an example to look for the optimal combination of $\mathrm{n}$ and P. Using 1-minute price data from Dec. 22th to Jan. 20th to train this trading model and get the optimal parameters. The result is shown in Figure 3.

We can see clearly that the optimal combination of $n$ and $\mathrm{P}$ for this commodity is to take the first 18 transaction days' average range and use 0.17 of this value as the

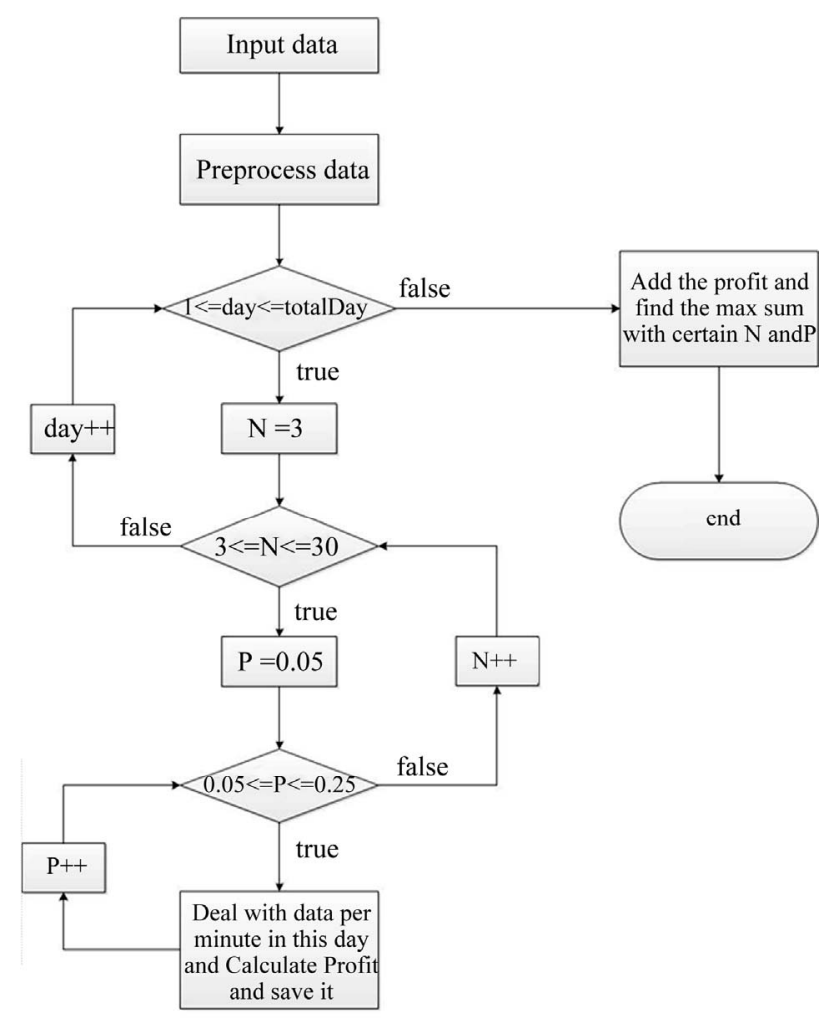

Figure 2. Algorithm flow chart. 
distance between $\mathrm{A}$ and the opening range.

These optimal parameters are to be used to determine the entry points for long and short of the next day, Jan. 30th. Program will adjust these parameters automatically through searching the optimal combination from the former 20 days and calculate the specific place of A and $\mathrm{C}$ for the next day constantly. Accumulative total profit under this trading strategy from Jan. 30th to March 21st is $2.02 \%$ and the number of trade is 36 in total, 19 for long and 17 for short. Daily profits in these days are shown in Figure 4. Given 0.5\% unilateral commission for stock index futures trading, net profit is $1.66 \%$. Taking the 5 - 6 times leverage level in Chinese stock index futures market, actual profits for investors can reach about $10 \%$ in 36 days.

Vast experiments on data shows that futures with large volumes, high-frequency turnovers and good fluidity suit this strategy better, for it reduces possibility of jumps (See Table 1). With less jumps, the price range and trend established during the first few minutes of market open can sets the tone for the rest of the day, making high and

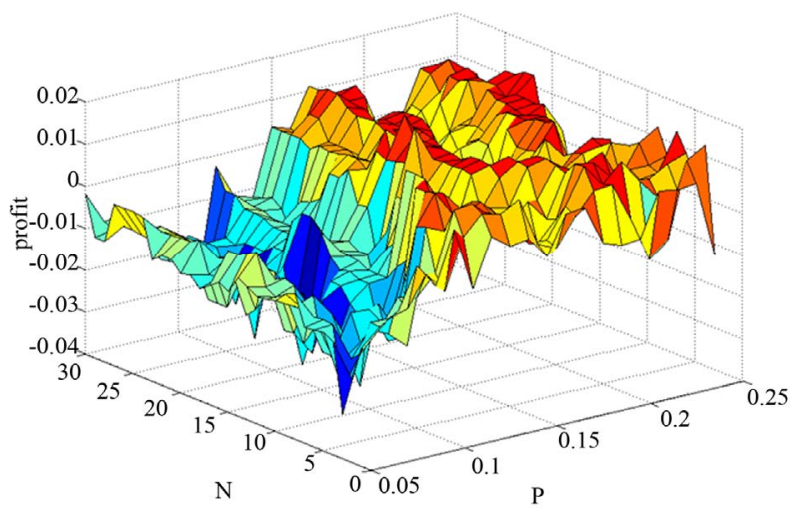

Figure 3. 3-dimentional accumulative profits for parameter optimization.

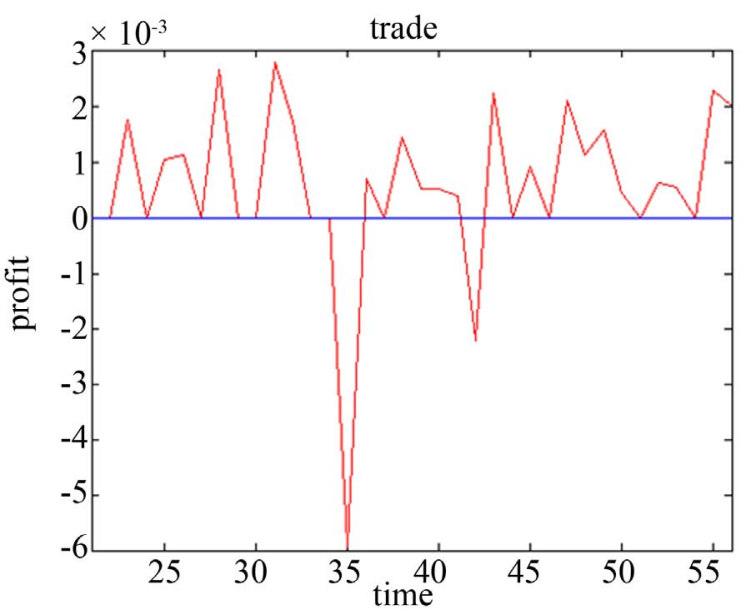

Figure 4. Daily profits of IF1206 from Jan. 30th to March 21st. low price for the full day already observable, with much greater likelihood than chance. We expand this trading strategy to the front month contract and the first back month contract in recent commodity futures market. Table 2 shows accumulative total profit from Jan. 19th to Apr. 19th and Table 3 shows accumulative total profit from Apr. 9th to May. 2nd.

Table 1. Trading details of IF1206.

\begin{tabular}{|c|c|c|c|c|c|c|}
\hline date & profit & $\mathbf{n}$ & $\mathbf{P}$ & long & short & total \\
\hline 2012/1/30 & 0 & 15 & 0.15 & 0 & 0 & 0 \\
\hline 2012/1/31 & 0 & 15 & 0.15 & 0 & 0 & 0 \\
\hline $2012 / 2 / 03$ & 0.001737 & 15 & 0.15 & 1 & 0 & 1 \\
\hline 2012/2/06 & 0 & 15 & 0.15 & 0 & 0 & 0 \\
\hline 2012/2/07 & 0.001035 & 15 & 0.15 & 0 & 1 & 1 \\
\hline $2012 / 2 / 08$ & 0.001112 & 15 & 0.15 & 1 & 0 & 1 \\
\hline $2012 / 2 / 09$ & 0 & 9 & 0.25 & 0 & 0 & 0 \\
\hline $2012 / 2 / 10$ & 0.002632 & 9 & 0.25 & 2 & 0 & 2 \\
\hline $2012 / 2 / 13$ & 0 & 9 & 0.25 & 0 & 0 & 0 \\
\hline $2012 / 2 / 14$ & 0 & 9 & 0.25 & 0 & 0 & 0 \\
\hline $2012 / 2 / 15$ & 0.002789 & 9 & 0.25 & 3 & 0 & 3 \\
\hline $2012 / 2 / 16$ & 0.001727 & 9 & 0.25 & 0 & 1 & 1 \\
\hline 2012/2/17 & 0 & 9 & 0.25 & 0 & 0 & 0 \\
\hline $2012 / 2 / 20$ & 0 & 9 & 0.25 & 0 & 0 & 0 \\
\hline $2012 / 2 / 21$ & -0.00593 & 9 & 0.25 & 0 & 2 & 2 \\
\hline $2012 / 2 / 22$ & 0.000687 & 13 & 0.18 & 1 & 0 & 1 \\
\hline $2012 / 2 / 23$ & 0 & 13 & 0.18 & 0 & 0 & 0 \\
\hline $2012 / 2 / 24$ & 0.001429 & 13 & 0.18 & 2 & 0 & 2 \\
\hline $2012 / 2 / 27$ & 0.000515 & 13 & 0.18 & 1 & 0 & 1 \\
\hline $2012 / 2 / 28$ & 0.000519 & 13 & 0.18 & 0 & 1 & 1 \\
\hline $2012 / 2 / 29$ & 0.000373 & 13 & 0.18 & 0 & 1 & 1 \\
\hline 2012/3/01 & -0.00223 & 13 & 0.18 & 2 & 0 & 2 \\
\hline 2012/3/02 & 0.002227 & 13 & 0.18 & 2 & 0 & 2 \\
\hline 2012/3/05 & 0 & 13 & 0.18 & 0 & 0 & 0 \\
\hline $2012 / 3 / 06$ & 0.000896 & 13 & 0.18 & 0 & 1 & 1 \\
\hline 2012/3/07 & 0 & 13 & 0.18 & 0 & 0 & 0 \\
\hline 2012/3/08 & 0.002104 & 13 & 0.18 & 2 & 0 & 2 \\
\hline 2012/3/09 & 0.001116 & 15 & 0.17 & 1 & 0 & 1 \\
\hline 2012/3/12 & 0.00157 & 15 & 0.17 & 0 & 2 & 2 \\
\hline 2012/3/13 & 0.000445 & 15 & 0.17 & 1 & 0 & 1 \\
\hline $2012 / 3 / 14$ & 0 & 12 & 0.17 & 0 & 0 & 0 \\
\hline $2012 / 3 / 15$ & 0.000608 & 12 & 0.17 & 0 & 1 & 1 \\
\hline 2012/3/16 & 0.000535 & 4 & 0.18 & 0 & 1 & 1 \\
\hline 2012/3/19 & 0 & 4 & 0.18 & 0 & 0 & 0 \\
\hline $2012 / 3 / 20$ & 0.002281 & 4 & 0.18 & 0 & 3 & 3 \\
\hline $2012 / 3 / 21$ & 0.001986 & 4 & 0.18 & 0 & 3 & 3 \\
\hline
\end{tabular}


Table 2. Total profit from Jan. 19th to Apr. 19th.

\begin{tabular}{ccccc}
\hline & profit & long & short & total \\
\hline L1205 & 0.0135 & 15 & 23 & 38 \\
RU1205 & 0.0019 & 12 & 13 & 25 \\
CF1205 & 0.0125 & 17 & 11 & 28 \\
TA1205 & -0.0056 & 17 & 12 & 29 \\
SR1209 & 0.0041 & 13 & 14 & 27 \\
AU1206 & -0.0028 & 19 & 10 & 29 \\
\hline
\end{tabular}

Table 3. Total profit from Mar. 8th to May. 2nd.

\begin{tabular}{ccccc}
\hline & profit & long & short & total \\
\hline PVC1209 & 0.0093 & 1 & 5 & 6 \\
M1209 & -0.0015 & 3 & 3 & 6 \\
Y1209 & 0.0014 & 2 & 2 & 4 \\
CU1206 & 0.0006 & 4 & 4 & 8 \\
ZN1206 & 0.0062 & 5 & 3 & 8 \\
RB1210 & 0.0067 & 1 & 9 & 10 \\
WS1209 & 0.0169 & 0 & 9 & 9 \\
WT1209 & 0.0085 & 5 & 10 & 15 \\
C1209 & 0.0029 & 4 & 10 & 14 \\
P1209 & 0.0039 & 1 & 2 & 3 \\
\hline
\end{tabular}

Empirical studies on Chinese stock index futures market have verified effectiveness of this improvement on this trading strategy because of the higher liquidity and lower commissions. But when transfer it to commodity futures market, since commissions in this market is relatively higher, about $5 \%$, net profits are not optimistic for many futures, at least in recent Chinese market.

Also in research, we find that the number of previous transaction days we take and the percent of the value we use are related with the number of trading. The shorter time and smaller value percent we use, the more trading we do. So the possibility of getting profits increase. However, we should determine the parameter combinations we use taking commissions into consideration. If it is relatively high, investors should be cautious to open positions, ensuring to balance the profits and costs. Longer time and larger value percent are recommended. The opposite situation also holds.

Those equities that work best for ACD are highly volatile, very liquid (lots of daily trading volume), and subject to long trends. Moreover, we will expand this method to ETF funds, foreign exchange and other financial derivatives which have good fluidity and high-frequency turnovers in Chinese market to get further conclusions of this trading strategy.

\section{Conclusion}

In this paper, we suggest an improvement on a famous trading strategy based on ACD rules and pivot point system, which was proposed by Mark B. Fisher, to futures market in China. Among studies concerning this strategy, discussions on the calculating method of the place of entry point $\mathrm{A}$ and $\mathrm{C}$ are rare. We expand this study, focusing on finding the optimal combination of parameters for entry point A. In program, we select every possible combination of the number of past transaction days we take to get average range, namely $\mathrm{n}$, and the percent value $P$, to get accumulate profits under each. For each day, program will train the model by high frequency data of the former 20 days, getting an optimal combination of parameters for the next transaction day, to determine the distance of A point away the bounds of opening range. This process will be repeated before market open each day automatically by program to adjust optimal parameters constantly. Empirical studies of this trading strategy in recent Chinese stock index futures market achieve substantial profits and verify its effectiveness, given to its high liquidity and low commissions. While in Chinese commodity futures market it fails to achieve sustainable net profits for most kinds in recent market conditions. Given the ineffectiveness in recent Chinese commodity futures market, investors should be cautious to apply this trading strategy. We will expand our study as Chinese futures market is maturing and having higher volumes and liquidity to get further conclusion about its effectiveness.

\section{REFERENCES}

[1] W. Zhuo, X. Zhao, Z. Zhou and S. Wang, "Study on Stock Index Futures Mean Reversion Effect and Arbitrage in China Based on High-Frequency Data," iBusiness, Vol. 4, No. 1, 2012, pp. 78-83. doi:10.4236/jilsa.2011.32010

[2] J. L. Bickford, "Forex Wave Theory-A Technical Analysis for Spot and Futures Currency Traders," The McGraw-Hill Companies, New York, 2007.

[3] K. Assaleh, H. El-Baz and S. Al-Salkhadi, "Predicting Stock Prices Using Polynomial Classifiers: The Case of Dubai Financial Market," Journal of Intelligent Learning Systems and Applications, Vol. 3, No. 2, 2011, pp. 82-89.

[4] Q. Qin, Q. Wang, S. Ge and G. Ramakrishnan, "Chinese Stock Price and Volatility Predictions with Multiple Technical Indicators," Journal of Intelligent Learning Systems and Applications, Vol. 3, No. 4, 2011, pp. 209-219. doi:10.4236/jilsa.2011.34024

[5] A. Hossain and M. Nasser, "Recurrent Support and Relevance Vector Machines Based Model with Application to Forecasting Volatility of Financial Returns," Journal of Intelligent Learning Systems and Applications, Vol. 3, No. 4, 2011, pp. 230-241. doi:10.4236/jilsa.2011.34026 
[6] V. Pacelli, V. Bevilacqua and M. Azzollini, "An Artificial Neural Network Model to Forecast Exchange Rates," Journal of Intelligent Learning Systems and Applications, Vol. 3, No. 2, 2011, pp. 57-69. doi:10.4236/jilsa.2011.32008

[7] V. Pacelli and M. Azzollini, “An Artificial Neural Network Approach for Credit Risk Management," Journal of Intelligent Learning Systems and Applications, Vol. 3, No. 2, 2011, pp. 103-112.

[8] V. Pacelli, "Pricing in Banking and Finance by Utilizing Artificial Neural Networks," In: R. W. Nelson, Ed., New Developments in Artificial Neural Network Research, Nova Science Publishers, Inc., New York, 2011.

[9] M. B. Fisher, "The Logical Trader," John Wiley \& Sons, Inc., Hoboken, 2002.

[10] M. Blackman, "Spotting Breakouts As Easy As ACD,"
2004.

http://www.investopedia.com/articles/technical/04/03240 4.asp

[11] I. Toshchakov, "Beat the Odds in Forex Trading," John Wiley \& Sons, Inc., Hoboken, 2006.

[12] J. L. Person, "Candlestick and Pivot Point Trading Triggers," John Wiley \& Sons, Hoboken, 2007.

[13] M. Blackman, "Market Reversals and How to Spot Them," 2004.

http://www.investopedia.com/articles/technical/04/03100 4.asp

[14] Z. W. Huang, "Empirical Evidence in Stock Index Futures Intraday Trading Based on ACD Rules and Pivot Point System," Guosen Report, 2010. 